\title{
NOTE ON THREE-DIMENSIONAL LIE GROUPS
}

\author{
by E. M. PATTERSON \\ (Received 15th July, 1953)
}

The object of this note is to construct a set of real three-dimensional Lie groups such that every real three-dimensional Lie group is locally isomorphic with some group in the set. The construction is effected by first finding canonical forms for the constants of structure of real three-dimensional Lie algebras ; these canonical forms give rise to certain bilinear forms, and the Lie groups are obtained as linear groups isomorphic with groups of automorphisms which leave these bilinear forms invariant.

In the case of complex three-dimensional Lie groups a similar construction is possible.

1. Lie algebras over a three-dimensional vector space.

Let $\mathbf{K}$ be a commutative field, $\mathbf{V}_{3}(\mathbf{K})$ a three-dimensional vector space over $\mathbf{K}$, and $\mathbf{L}$ a Lie algebra $\dagger$ over $\mathbf{V}_{3}(\mathbf{K})$ having structural constants $\ddagger c_{j k}^{i}$ with respect to a basis $\mathbf{e}_{i}$. Let $A$ be the $3 \times 3$ matrix $\left(a^{i j}\right)$ defined by

$$
a^{i j}={ }_{2}^{1} \epsilon^{i p q} c_{p q}^{j},
$$

where $\epsilon^{i p q}\left(=\epsilon_{i p q}\right)$ are the usual permutation symbols. The structural constants satisfy§

$$
\begin{array}{r}
c_{j k}^{i}+c_{k j}^{i}=0, \ldots \ldots . \\
c_{i q}^{p} c_{j k}^{q}+c_{j q}^{p} c_{k i}^{q}+c_{k q}^{p} c_{i j}^{q}=0 .
\end{array}
$$

Equations (2) impose no restriction on the matrix $A$, on account of the skew-symmetry of the permutation symbols. Calculation shows that equations (3) are satisfied if and only if the adjoint of $A$ is symmetric. Conversely, given any $3 \times 3$ matrix $A$ whose adjoint is symmetric, the constants $c_{j k}^{i}$ defined by

$$
c_{j k}^{i}=\epsilon_{p j k} a^{p i}
$$

satisfy (2) and (3), and hence determine a Lie algebra over $\mathbf{V}_{3}(\mathbf{K})$. Therefore Lie algebras over $\mathbf{V}_{\mathbf{3}}(\mathbf{K})$ are in one-one correspondence with $3 \times 3$ matrices over $\mathbf{K}$ whose adjoints are symmetric.

Theorem 1. Two lie algebras $\mathbf{L}, \mathrm{L}^{*}$ over $\mathbf{V}_{3}(\mathbf{K})$, whose constants of structure are represented by the matrices $A, A^{*}$ in terms of some basis, are isomorphic if and only if there is a non-singular $3 \times 3$ matrix $P$ over $\mathbf{K}$ such that $\|$

$$
|P| A^{*}=P^{\prime} A P \text {. }
$$

It is clear that $L$ and $L^{*}$ are isomorphic if and only if there are bases for $V_{3}(\mathbf{K})$ in terms of which the constants of structure are identical. Let $\mathbf{e}_{i}, \mathrm{e}_{i}^{*}$ be two bases for $\mathbf{V}_{3}(\mathbf{K})$. Then there are elements $p_{i}^{j}$ of $\mathbf{K}$ such that $\mathrm{e}_{i}=p_{i}^{j} \mathrm{e}_{j}^{*}$, where the matrix $P=\left(p_{i}^{j}\right)$ is non-singular. Suppose that $A, A^{*}$ represent the constants of structure of the algebras $\mathrm{L}, \mathrm{L}^{*}$ in terms of the basis $\mathrm{e}_{i}$. Since the structural constants of a Lie algebra transform as components of a tensor of covariant order two and contravariant order one, and the permutation symbols $\epsilon^{i j k}$ transform as

† See, for example, C. Chevalley, "Theory of Lie groups" (Princeton University press), Chapter IV. used.

$\ddagger$ The suffixes $i, j, k, p, q$ take the values $1,2,3$ and the summation convention for repeated indices is

$\S$ Chevalley, loc. cit.

$\||P|$ denotes the determinant of $P$, and $P^{\prime}$ denotes the transpose of $P$. 
components of a third order contravariant tensor of weight -1 , the elements $a^{i j}$ of the matrix $A$ transform as components of a second order contravariant tensor of weight -1 . Hence $\mathrm{L}$ is represented by $A^{*}$ in terms of the basis $\mathrm{e}_{i}$ if and only if $|P| A^{*}=P^{\prime} A P$. Theorem 1 is therefore proved.

2. Canonical forms for the constants of structure of real three-dimensional Lie algebras.

Two matrices $A, A^{*}$ satisfying (5) for some non-singular matrix $P$ will be called quasicongruent. It is easily shown that quasi-congruence is a proper equivalence relation, and that it preserves rank, symmetry, skew-symmetry and symmetry of the adjoint matrix. Canonical forms for the constants of structure of three-dimensional Lie algebras can therefore be obtained by finding canonical forms for quasi-congruence classes of $3 \times 3$ matrices whose adjoints are symmetric. This can easily be done for the real and complex fields ; in the remainder of this paper only the real case will be considered.

Lemma. Two real $3 \times 3$ matrices $A, A^{*}$ are quasi-congruent if and only if either (i) $A$ is congruent to $A^{*}$ or (ii) $-A$ is congruent to $A^{*}$.

Suppose that $A$ and $A^{*}$ are real $3 \times 3$ matrices satisfying (5) for some real non-singular $3 \times 3$ matrix $P$. If $|P|>0$, put $Q=P / \sqrt{ }|P|$; then $A^{*}=Q^{\prime} A Q$ and so $A$ and $A^{*}$ are congruent. If $P \mid<0$, put $Q=P / \sqrt{ }(-|P|)$; then $A^{*}=-Q^{\prime} A Q$, and so $-A$ and $A^{*}$ are congruent. Conversely, if $A$ and $A^{*}$ satisfy $A^{*}=Q^{\prime} A Q$ for some non-singular matrix $Q$, then $|P| A^{*}=P^{\prime} A P$, where $P=Q /|Q|$, so that $A$ and $A^{*}$ are quasi-congruent. Similarly, if - $A$ and $A^{*}$ are congruent, then $A$ and $A^{*}$ are quasi-congruent.

Theorem 2. Every Lie Algebra over a real three-dimensional vector space is isomorphic with one of the following algebras :

$$
\begin{array}{llllll}
L_{1}: c_{23}^{1}=1 & c_{31}^{2}=1 & c_{12}^{3}=1 & \\
L_{2}: c_{23}^{1}=1 & c_{31}^{2}=1 & c_{12}^{3}=-1 & \\
L_{3}(\alpha): & c_{23}^{1}=1 & c_{23}^{2}=\alpha & c_{31}^{2}=1 & (\alpha \geqslant 0) \\
L_{4}(\alpha): & c_{23}^{1}=1 & c_{23}^{2}=\alpha & c_{31}^{2}=-1 & (\alpha \geqslant 0) \\
L_{5}: c_{23}^{2}=1 & c_{31}^{1}=-1 & & \\
L_{6}: c_{23}^{1}=1 & & & \\
L_{7}: & c_{23}^{2}=1 & & & \\
L_{8}: \text { the zero algebra } & & &
\end{array}
$$

(in each case the remaining components $c_{i j}^{k}$ except those related by (2) are zero).

From Theorem 1 and the lemma, it follows that two Lie algebras $\mathbf{L}, \mathbf{L}^{*}$ over a real threedimensional vector space are isomorphic if and only if either $A$ and $A^{*}$ are congruent or $-A$ and $A^{*}$ are congruent. Consequently to each set of isomorphic Lie algebras there corresponds a class of matrices, whose adjoints are symmetric, each congruent to a given $3 \times 3$ matrix or its negative. Canonical forms for such classes are readily found by using the usual elementary operations. If $A$ is non-singular, and its adjoint is symmetric, then $A$ itself is symmetric. The canonical matrices in this case are therefore

$$
C_{1}=I=\left(\begin{array}{ccc}
1 & . & \cdot \\
\cdot & 1 & . \\
. & . & 1
\end{array}\right), \quad C_{2}=\left(\begin{array}{ccc}
1 & . & \cdot \\
\cdot & 1 & \cdot \\
. & . & -1
\end{array}\right) \text {. }
$$

If $A$ is singular, the symmetry of the adjoint matrix does not imply that $A$ is symmetric. However the canonical forms can easily be found to be 


$$
\begin{aligned}
& C_{3}(\alpha)=\left(\begin{array}{lll}
1 & \alpha & \cdot \\
. & 1 & . \\
. & . & .
\end{array}\right)(\alpha \geqslant 0), \quad C_{4}(\alpha)=\left(\begin{array}{rrr}
1 & \alpha & . \\
\cdot & -1 & . \\
. & . & .
\end{array}\right)(\alpha \geqslant 0), \\
& C_{5}=\left(\begin{array}{rrr}
\cdot & 1 & \cdot \\
-1 & \cdot & \cdot \\
\cdot & \cdot & \cdot
\end{array}\right), \quad C_{6}=\left(\begin{array}{lll}
1 & \cdot & \cdot \\
\cdot & \cdot & \cdot \\
\cdot & \cdot & .
\end{array}\right), \quad C_{7}=\left(\begin{array}{lll}
\cdot & 1 & \cdot \\
\cdot & \cdot & \cdot \\
. & \cdot & .
\end{array}\right), C_{8}=0 .
\end{aligned}
$$

In the cases of $C_{3}(\alpha)$ and $C_{4}(\alpha)$, there is one canonical matrix for each non-negative value of $\alpha$. No two of the matrices in the set are quasi-congruent. The corresponding Lie algebras are precisely those which are listed in the statement of Theorem 2 , which is therefore proved.

The canonical matrices $C_{1}, \ldots, C_{8}$ give rise to the following bilinear forms, which correspond respectively to the Lie algebras $L_{1}, \ldots, L_{8}$ :

$$
\begin{aligned}
\mathbf{f}_{1} & \equiv x_{1} x_{2}+y_{1} y_{2}+z_{1} z_{2}, \quad \mathbf{f}_{2} \equiv x_{1} x_{2}+y_{1} y_{2}-z_{1} z_{2}, \\
\mathbf{f}_{3}(\alpha) & \equiv x_{1} x_{2}+\alpha x_{1} y_{2}+y_{1} y_{2}, \quad \mathbf{f}_{4}(\alpha) \equiv x_{1} x_{2}+\alpha x_{1} y_{2}-y_{1} y_{2}, \\
\mathbf{f}_{5} & \equiv x_{1} y_{2}-y_{1} x_{2}, \quad \mathbf{f}_{6} \equiv x_{1} x_{2}, \quad \mathbf{f}_{7} \equiv x_{1} y_{2}, \quad \mathbf{f}_{8} \equiv 0 .
\end{aligned}
$$

\section{Real three-dimensional Lie groups.}

Let $\mathrm{L}$ be a Lie algebra over a real three-dimensional vector space, and let $A$ be the corresponding matrix with respect to some basis. From equation (5) an automorphism of the vector space, leaving this matrix unaltered, is represented by a non-singular matrix $P$ such that

$$
|P| A=P^{\prime} A P \text {. }
$$

The set of all non-singular matrices $P$ satisfying (6) for a fixed matrix $A$ forms a group, which will be denoted by $\mathrm{G}(A)$. A simple argument, similar to that used in proving the lemma in section 2, shows that this group is isomorphic with the group of matrices $Q$ such that $A=Q^{\prime} A Q$ and $|Q|>0$; hence $\mathrm{G}(A)$ is isomorphic with a subgroup of the group of automorphisms leaving the bilinear form $a^{i j} x_{i} y_{j}$ invariant.

In particular, $\mathbf{G}\left(C_{1}\right), \ldots, \mathbf{G}\left(C_{8}\right)$ are isomorphic with subgroups of the groups of automorphisms leaving $f_{1}, \ldots, \mathbf{f}_{8}$ respectively invariant. The groups $\mathbf{G}\left(C_{1}\right)$ and $\mathbf{G}\left(C_{2}\right)$ are in fact the well-known "rotation groups". Direct calculations show that the remaining groups can be represented by matrices of the following forms :

$$
\begin{aligned}
& \mathbf{G}\left\{C_{3}(\alpha)\right\} \text { by }\left(\begin{array}{ccc}
a & -b & 0 \\
b & a+\alpha b & 0 \\
c & d & 1
\end{array}\right) \text {, where } a^{2}+b^{2}+\alpha a b \neq 0, \\
& \mathbf{G}\left\{C_{4}(\alpha)\right\} \text { by }\left(\begin{array}{ccc}
a & b & 0 \\
b & a+\alpha b & 0 \\
c & d & 1
\end{array}\right) \text {, where } a^{2}-b^{2}+\alpha a b \neq 0 \text {, } \\
& \mathbf{G}\left(C_{5}\right) \text { by }\left(\begin{array}{lll}
a & d & 0 \\
b & e & 0 \\
c & f & 1
\end{array}\right) \text {, where } a e \neq b d \text {, } \\
& \mathbf{G}\left(C_{6}\right) \text { by }\left(\begin{array}{ccc}
c f-e d & 0 & 0 \\
a & c & e \\
b & d & f
\end{array}\right) \text {, where } c f \neq d e \\
& \mathbf{G}\left(C_{7}\right) \text { by }\left(\begin{array}{lll}
a & 0 & 0 \\
0 & c & 0 \\
b & d & 1
\end{array}\right) \text {, where } a c \neq 0 \\
& \mathbf{G}\left(C_{8}\right) \text { by arbitrary non-singular } 3 \times 3 \text { matrices. }
\end{aligned}
$$


In passing, we observe that $\mathbf{G}\left\{C_{3}(\alpha)\right\}, \mathbf{G}\left\{C_{4}(\alpha)\right\}$ and $\mathbf{G}\left(C_{7}\right)$ are all subgroups of $\mathbf{G}\left(C_{5}\right)$; also that $\mathbf{G}\left\{C_{3}(\alpha)\right\}(\alpha \neq 2)$ is isomorphic with a subgroup of $\mathbf{G}\left\{C_{3}(0)\right\}$, that $\mathbf{G}\left\{C_{4}(\alpha)\right\}$ is isomorphic with a subgroup of $\mathbf{G}\left\{C_{4}(0)\right\}$ and that $\mathbf{G}\left\{C_{3}(2)\right\}$ is isomorphic with a subgroup of $\mathrm{G}\left(C_{8}\right)$. Most of these relationships follow from the facts that, if $P \in \mathbf{G}(A)$, then $P \in \mathbf{G}\left(A+A^{\prime}\right)$ and $P \in \mathbf{G}\left(A-A^{\prime}\right)$.

The groups $\mathbf{G}\left(C_{1}\right), \ldots, \mathbf{G}\left(C_{8}\right)$ are all Lie groups; their Lie algebras contain $\mathbf{L}_{1}, \ldots, \mathbf{L}_{8}$ respectively as sub-algebras. To find Lie groups having $\mathbf{L}_{1}, \ldots, \mathbf{L}_{8}$ as $\mathrm{Lie}$ algebras we therefore find appropriate subgroups of $\mathbf{G}\left(C_{1}\right), \ldots, \mathbf{G}\left(C_{8}\right)$. Such subgroups can be found by inspection. The funal results can be obtained in the following form :

$\mathbf{G}\left(C_{1}\right), \mathbf{G}\left(C_{2}\right)$ have Lie algebras $\mathbf{L}_{1}, \mathbf{L}_{2}$ respectively. The group $\mathbf{H}_{3}(\alpha)$ of matrices of the form

$$
\left(\begin{array}{ccc}
f^{\prime}(z) & f(z) & 0 \\
-f(z) & f^{\prime}(z)-\alpha f(z) & 0 \\
y & x & 1
\end{array}\right)
$$

where $f(z)=\beta^{-1} \exp \left(\frac{1}{2} \alpha z\right) \sinh (\beta z)\left(\beta=\frac{1}{2} \sqrt{\alpha^{2}-4}\right)$ if $\alpha>2, f(z)=z \exp z$ if $\alpha=2$ and $f(z)=$ $\gamma^{-1} \exp \left(\frac{1}{2} \alpha z\right) \sin (\gamma z)\left(\gamma=\frac{1}{2} \sqrt{4-\alpha^{2}}\right)$ if $0 \leqslant \alpha<2$, has $\mathbf{L}_{3}(\alpha)$ as its Lie algebra. The group $\mathbf{H}_{4}(\alpha)$ of matrices of the form

$$
\left(\begin{array}{ccc}
f^{\prime}(z)-\alpha f(z) & f(z) & 0 \\
f(z) & f^{\prime}(z) & 0 \\
x & y & 1
\end{array}\right)
$$

where $f(z)=\delta^{-1} \exp \left(\frac{1}{2} \alpha z\right) \sinh (\delta z)\left(\delta=\frac{1}{2} \sqrt{\alpha^{2}+4}\right)$, has $\mathbf{L}_{4}(\alpha)$ as its Lie algebra. The groups $\mathbf{H}_{5}, \mathbf{H}_{6}, \mathbf{H}_{7}$ and $\mathbf{H}_{8}$ of matrices of the forms

$$
\begin{aligned}
& \left(\begin{array}{ccc}
z+1 & 0 & 0 \\
0 & z+1 & 0 \\
y & x & 1
\end{array}\right), z \neq-1, \\
& \left(\begin{array}{ccc}
1 & 0 & 0 \\
z & 1 & 0 \\
x & y & 1
\end{array}\right), \\
& \left(\begin{array}{ccc}
1+z & 0 & 0 \\
y & 1 & x \\
0 & 0 & 1
\end{array}\right), z \neq-1, \\
& \left(\begin{array}{ccc}
x & 0 & 0 \\
0 & y & 0 \\
0 & 0 & z
\end{array}\right), x y z \neq 0,
\end{aligned}
$$

have $L_{5}, L_{6}, L_{7}$ and $L_{8}$ respectively as their Lie algebras. Using these results and Theorem 2, we obtain

TheOREM 3. Every real three-dimensional Lie group is locally isomorphic with one of the groups $\mathbf{G}\left(C_{1}\right), \mathbf{G}\left(C_{2}\right), \mathbf{H}_{3}(\alpha), \mathbf{H}_{4}(\alpha), \mathbf{H}_{5}, \mathbf{H}_{6}, \mathbf{H}_{7}$ and $\mathbf{H}_{8}$.

UNITED COLLEGE,

UNIVERSITY OF ST. ANDREWS. 